\title{
EPSL
}

Earth and Planetary Science Letters 162 (1998) 15-25

\section{The influences of surface temperature on upwellings in planetary convection with phase transitions}

\author{
Volker Steinbach ${ }^{\mathrm{a}, *}$, David A. Yuen ${ }^{\mathrm{b}}$ \\ ${ }^{a}$ Inst. f. Theoretical Geophysics, Earth Science Institute, University of Utrecht, Budapestlaan 4, 3508 TA Utrecht, The Netherlands \\ ${ }^{b}$ Minnesota Supercomputer Institute and Department of Geology and Geophysics, University of Minnesota, Minneapolis, \\ MN 55415-1227, USA
}

Received 21 February 1998; revised version received 17 June 1998; accepted 1 July 1998

\begin{abstract}
The importance of surface temperature for mantle convection appears with the presence of adiabatic heating and cooling and the release and consumption of latent heat in the presence of phase transitions. For some planetary bodies these effects cannot be neglected. The dimensionless surface temperature $T_{0}$, which is the ratio between the temperature at the top of the convective region and the temperature drop across the mantle, is close to one for Mars and Venus. For the Earth, $T_{0}$ lies between 0.2 and 0.5 . The dynamical influence of $T_{0}$ is especially poignant for internally heated convection with temperature-dependent viscosity. There is a tight coupling between the magnitude of the temperature field and the viscosity itself. We have studied temperature-dependent viscosity convection for both low- $T_{0}(0.2)$ and high- $T_{0}(1.2)$ situations and with internal heating in mantle convection with two upper-mantle phase transitions. Our results show that within this range of $T_{0}$ there exist two regimes for the evolution of upwellings in the mantle. In transient situations plume-plume collisions lead to the formation of megaplumes for high- $T_{0}$ regimes but are less likely to do so for low $T_{0}$. In the long-term regime, plumes with low $T_{0}$ are prone to develop from the transition zone with a supply of hot material coming from the shallow lower mantle. In systems with high $T_{0}$, however, long-lived plumes tend to have deeper mantle origins. In quasi-layered situations high $T_{0}$ may act as a positive feed-back mechanism in inducing powerful hot upwellings into the upper mantle. (C) 1998 Elsevier Science B.V. All rights reserved.
\end{abstract}

Keywords: convection; Earth; Venus; mantle plumes; phase transitions

\section{Introduction}

In mantle convection with adiabatic heating present, the effects of surface temperature come into play. This point, however, has not been generally recognized in the geodynamical community, except for the early work on linear stability by Peltier [1]

\footnotetext{
* Corresponding author. Fax: +31 30253 5030;

E-mail: steiney@geo.uu.nl
}

and the later study by Steinbach [2]. Finite-amplitude studies on the influence of surface temperature on constant viscosity convection have been carried out by Steinbach [2], Bercovici et al. [3], Hansen and Yuen [4] and Yuen et al. [5]. The surface temperature is commonly expressed as a dimensionless number $T_{0}$, which represents the ratio between the temperature at the top of the convecting layer and the temperature drop across this layer. The quantity $T_{0}$ enters into the energy equation in the anelastic-liq- 
uid [6] and the extended Boussinesq approximation $[7,8]$, since the amount of adiabatic heat released or consumed by compression or expansion is proportional to the absolute temperature. Why is the magnitude of this particular parameter important for planetary convection? Its importance lies in its great variability. For the Earth's mantle we have taken into account that our model cannot simulate plate-like behavior by introducing a sublithospheric temperature, rather than the actual surface temperature into the model. Thus $T_{0}$ then lies between 0.2 and $0.5[6,9]$. For Venus and Mars, when one takes into account the rather thick lithosphere in these planets without plate tectonics [10], $T_{0}$ may reach values of around 1.0 and 1.3 respectively. For these planets the surface temperature would be associated with the value at the base of the lithosphere, where a small amount of creep takes place. For the Jovian moons, such as Europa and Io, $T_{0}$ may even be larger. For the Earth's outer core $T_{0}$ may range between 3 and 4 . In these systems the surface temperature is significant compared to the temperature difference across the convecting layer. Therefore, it is important to study the dynamical effects of large $T_{0}$, since the surface boundary condition definitely produces a different style of convection from the low- $T_{0}$ regime [3]. Until now, there has been no work done on the effects of high $T_{0}$ on convection with phase transitions and temperature-dependent viscosity. In the case of phase transitions, the surface temperature is even more important, since the amount of latent heat produced in phase transitions is directly proportional to the absolute temperature (under the assumption of an enhanced thermal expansivity [7] and see Eq. 3 below). Furthermore, the effects of internal heating and temperature-dependent viscosity can also interact with the magnitude of $T_{0}$. Our purpose in this paper is to investigate the effects of varying $T_{0}$ on upwellings in planetary convection with phase transitions for planets with size close to the Earth and Venus.

\section{Model description}

We use a two-dimensional cartesian model based on the extended Boussinesq approximation [7]. The effects of viscous dissipation and adiabatic heating and cooling are included as is the release and con- sumption of latent heat from the phase transitions. This approach, in a mathematical sense, emerges from the fully compressible anelastic-liquid approximation [6] in the limit $\gamma \rightarrow \infty$, where $\gamma$ is the thermodynamic Grueneisen parameter. Physically speaking, in this limit $(D / \gamma \rightarrow 0)$ density variations due to compression are neglected, whereas the thermal and buoyancy effects of compression and expansion are retained.

We have employed the vorticity-streamfunction approach for a fluid with temperature-dependent viscosity. There are three control parameters in the extended Boussinesq framework: the Rayleigh number $\mathrm{Ra}$, the surface dissipation number $D_{0}$ and the surface temperature $T_{0}$. The governing equations in the extended Boussinesq approximation are:

$\nabla^{2} \psi=\omega$

$\nabla^{2}(\eta \omega)=\bar{\alpha} \operatorname{Ra} T_{x}+2\left(\eta_{z z} \psi_{x x}-2 \eta_{x z} \psi_{x z}+\eta_{x x} \psi_{z z}\right)$

$\frac{D T}{D t}-\psi_{x} \bar{\alpha} D_{0}\left(T_{0}+T\right)=\nabla^{2} T+R+\frac{D_{0}}{\mathrm{Ra}} \Phi$

Here $\omega, \psi$ and $T$ denote vorticity, streamfunction and temperature, respectively, and $\Phi$ is the viscous dissipation function. As scaling factors for length, time and temperature, we used the depth of the layer, $h$, thermal diffusion time, $h^{2} / \kappa_{0}\left(\kappa_{0}\right.$ being the surface thermal diffusion coefficient) and the temperature drop across the layer, $\Delta T$, respectively. A dimensionless time of 0.001 corresponds to $267 \mathrm{Myr}$. Subscripts $x$ and $z$ indicate partial derivatives and $D / D t$ is the total time derivative in the Lagrangian sense. The $z$-axis points upwards. Boundary conditions are free-slip everywhere, constant temperature at top and bottom and adiabatic at the side-walls. We note that $T_{0}$ appears in the adiabatic heating term in Eq. 3 and thus, via the effective expansion coefficient $\bar{\alpha}$, also in the latent heat release and consumption.

We have considered a temperature-dependent viscosity of the (dimensionless) Arrhenius form

$H=\exp \left(\frac{A}{T_{1}+T}\right) \exp \left(-\frac{A}{T_{1}}\right)$

where the constant second factor on the right hand side normalizes the surface viscosity to 1 . In all experiments described in this paper, we used a value of 
$T_{1}=0.2$ and chose the second parameter $A$ such that the viscosity varied by a factor of 200 . We did not vary $T_{1}$ with varying surface temperature $T_{0}$, since we wished to investigate only the thermodynamical effects of $T_{0}$, without touching any rheological aspects.

The derivation of the non-dimensional equations governing convection with phase transitions has been provided in Steinbach and Yuen [11]. The effects of phase transitions are incorporated by an effective thermal expansivity $\bar{\alpha}[7,11]$. It consists of a depthdependent background portion, which decreases by a factor of seven across the mantle [12] and an additional 'phase-transition' part, which peaks in the phase-transition regions and is negative for the endothermic spinel $\rightarrow$ perovskite transformation. Clapeyron slopes of 3 and $-2.5 \mathrm{MPa} / \mathrm{K}$ and relative density changes of $8 \%$ and $10 \%$ were assumed for the olivine $\rightarrow$ spinel and spinel $\rightarrow$ perovskite transition, respectively.

$\mathrm{Ra}$ and $D_{0}$ denote the surface Rayleigh and surface dissipation number. The surface dissipation number $D_{0}$ is given by $\alpha_{0} g h / C_{\mathrm{p}}$, where $\alpha_{0}$ is the surface thermal expansivity, $C_{\mathrm{p}}$ is the heat capacity and $g$ the gravitational acceleration. We use (nondimensional) values of $D_{0}=0.5$ and $\mathrm{Ra}=5 \times 10^{5}$, which, with temperature-dependent viscosity, results in a bottom Rayleigh number of $10^{8}$. The two remaining parameters are the nondimensional surface temperature $T_{0}$ and the internal heating parameter $R$, which is given as the ratio of the Rayleigh number due to internal heat sources and the surface Rayleigh number. We consider both entirely bottom heated $(R=0)$ and internally heated models with $R=10$, which is close to a chondritic abundance of radiogenic heat sources and lies well within geophysical constraints [9]. The surface temperature is varied between 0.2 and 1.2. In this paper, only the end-members $\left(T_{0}=0.2\right.$ and $\left.T_{0}=1.2\right)$ are shown.

The model mantle is $2900 \mathrm{~km}$ deep with the endothermic and exothermic phase transitions at 660 $\mathrm{km}$ and $400 \mathrm{~km}$ depth, respectively. A half-width of $36 \mathrm{~km}$ has been used in the formulation of the effective thermal expansivity [11]. All calculations were carried out in a rectangular domain of aspect ratio 5 , which is covered by 52 (vertical) by 260 finite elements of first order for the temperature and second order for the streamfunction and vorticity.
Vertical mesh refinement was used to ensure that the lower and upper thermal boundary layer and both of the phase transition regions were covered by at least 4 elements or about $\mathrm{O}(10 \mathrm{~km})$ vertical resolution. We used a constant initial temperature field with error-function profiles in upper and lower thermal boundary and superimposed a perturbation of the form $0.01 \cos (21 \pi x) \sin (\pi z)$.

A streamline-upwind Petrov-Galerkin scheme together with a second-order predictor-corrector method was applied for the solution of the timedependent Eq. 3, while Eqs. 1 and 2 were solved with a Galerkin method. Since the streamfunction feeds back into the vorticity equation when variable viscosity is included, we used an overrelaxation method to iteratively solve the combined Eqs. 1 and 2. For this subiteration, we used a global convergence criterion:

$\varepsilon=\sqrt{\frac{\sum_{i=1}^{n}\left(\Psi_{l+1}-\Psi_{l}\right)^{2}}{\sum_{i=1}^{n} \Psi_{l+1}^{2}}}$

where $l+1$ is the number of subiterations and $n$ is the number of nodal points. Convergence is satisfied when $\varepsilon<10^{-5}$.

\section{Results}

First, we describe the early stages of the flows that evolve under purely basally heated $(R=0)$ conditions for both low- $\left(T_{0}=0.2\right)$ and high- $\left(T_{0}=1.2\right)$ surface temperatures. Both cases were started with the same initial temperature field described in Section 2. Due to this initial condition, convection sets in within 21 equally spaced low aspect ratio cells in the upper and lower mantle separately. Since this state is almost completely layered, the lower mantle heats up significantly while the upper mantle is efficiently cooled. This gives rise to a possibly highly unstable temperature stratification. One might argue that this situation is artificially forced by the initial conditions. There are, however, some suggestions that convection was much more layered in the early history of both Earth and Venus due to the 

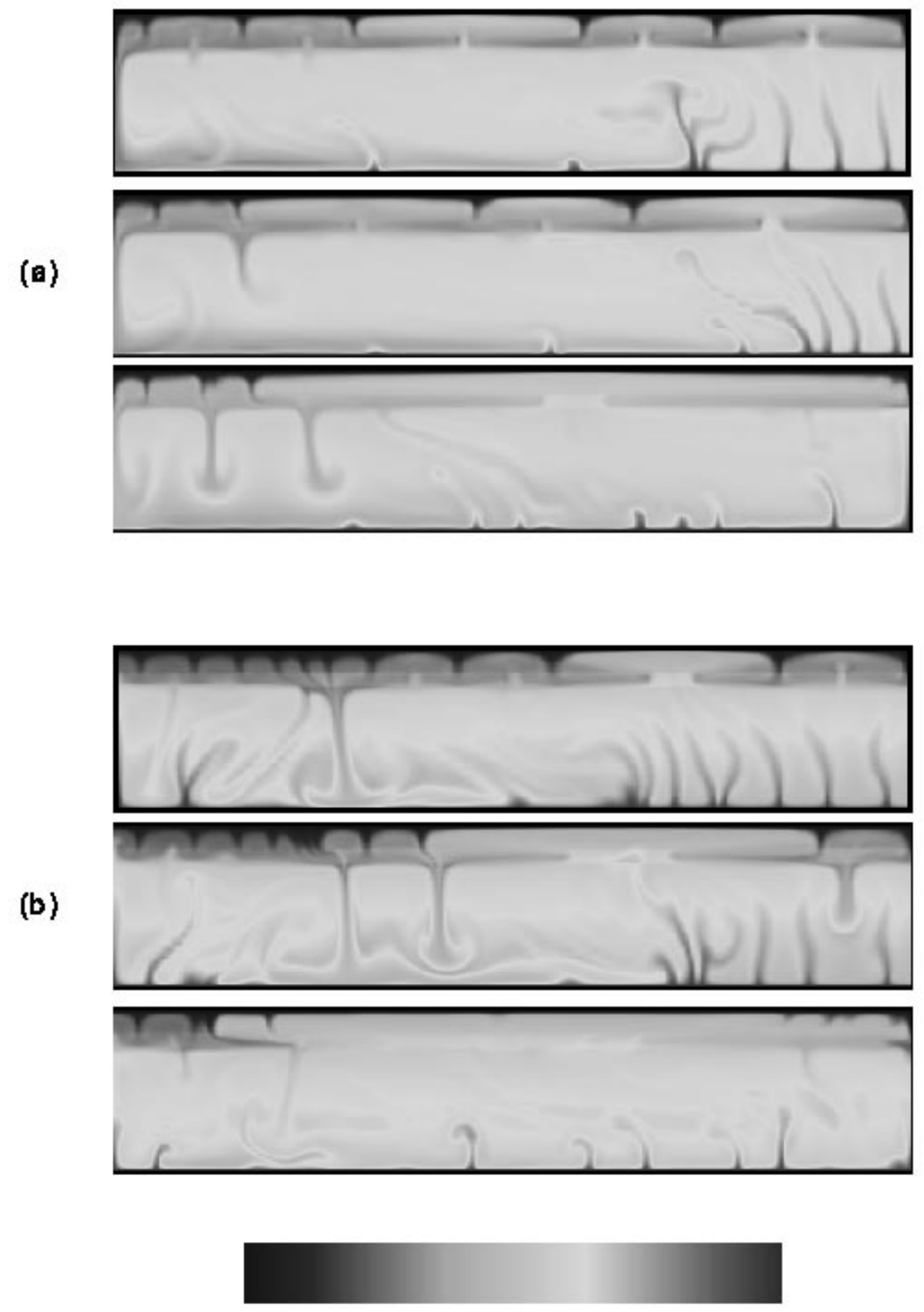
higher ambient temperatures and the greater abundance of radiogenic heat sources and, in fact, higher convective vigor [13-17]. Thus it is interesting to investigate the evolution of such a possibly unstable scenario. In Fig. 1 we show snapshots of the temperature distribution near the end of the layered state for low (a) and high (b) surface temperature. In the low- $T_{0}$ case, the mass exchange between upper and lower mantle that follows the initially layered state is largely dominated by an active broad downwelling (Fig. 1(a), upper and middle panel), while the return flow from lower to upper mantle is shallow: There is no continuous upward flow between the core-mantle boundary $(\mathrm{CMB})$ and the surface, a situation that has been observed in earlier simulations [18].

In the high- $T_{0}$ case, such a continuous upwelling is present (Fig. 1(b), upper panel). It is formed by the collective merging together of instabilities at the CMB. This reinforced form then penetrates the transition zone into the upper mantle. A collective merging phenomenon like this has been observed under different circumstances by Thompson and Tackley [19] and may be of interest for the past tectonics and resurfacing of Venus [20,21]. Because the magnitude of the latent heat released is proportional to $T_{0}$ [11], the increased amount of latent heat release in the high- $T_{0}$ case is illustrated by the comparatively high temperatures in the transition zone (Fig. 1(b), middle panel).

The late stage of the breakdown of layered convection is characterized in both cases by a massive upwelling of hot material from the shallow lower mantle into the upper mantle, where it spreads horizontally on a large scale (Fig. 1, lower panels).

The long-term development of the basally heated $(R=0)$ flows is illustrated in Fig. 2. Here snapshots of the residual temperature $\delta T=T-\langle T\rangle$ ( $\langle T\rangle$ being the horizontally averaged temperature) are shown. Time elapsed is around $3 \mathrm{Gyr}$ after the initial stage. As shown in the figure, flow structures in this late stage represent hybrid structures of layered and whole-mantle convection. Positive temperature anomalies in the upper mantle are more prominent in the low- $T_{0}$ case and vary in size from a few hundred to a few thousand kilometers (Fig. 2a, bottom panel). They are caused by intrusions of lower mantle material that are most often not connected to hot instabilities at the CMB but come from rather shallow lower mantle depths. The same is true for cold temperature anomalies in the lower mantle, which often seem to emerge from the transition zone and have lost connection to the cold surface instabilities.

On the other hand, the flow at high $T_{0}$ (Fig. 2b) exhibits cold temperature anomalies that are continuous from the surface to the CMB. In this case, the flow structure is much less complicated and is dominated by fewer downwellings. Again, most of the upwellings tend to merge into one dominant upwelling site. Intrusions of lower mantle material into the upper mantle may have both shallow or deep origin and are less voluminous than the large intrusions observed in the low- $T_{0}$ case.

In Fig. 3 snapshots of the residual temperature fields for the internally heated $(R=10)$ cases are shown for low (a) and high (b) surface temperature $T_{0}$. For low $T_{0}$, the upper mantle thermal structure is dominated by large hot temperature anomalies, which, again, have little or no connection to deep lower mantle sources. In the high- $T_{0}$ case, however, a very stable central plume has formed that penetrates the transition zone and rises straight up to the surface. Again, the flow structure is more complicated in the low- $T_{0}$ case; many boundary layer instabilities are suppressed by the higher rates of adiabatic heating and cooling for the high- $T_{0}$ situation.

In convection with temperature-dependent viscosity there is a distinct possibility for lubrication of the flow from the stress reduction due to hot anomalies, which locally reduces the viscosity [22]. To study this type of thermomechanical phenomenon, we have plotted cross sections of the residual temperature $\delta T$ and the associated magnitude of the shear stress $\log \left|\tau_{x z}\right|$. Here we concentrated on the regions of upwellings in the basally heated, low- $T_{0}$ case shown in Fig. 2. Isolated hot subsurface anoma-

Fig. 1. Snapshots of the temperature field in the early stages of the base heated flows. (a) Low surface temperature $\left(T_{0}=0.2\right)$. Time intervals between second and first and between third and first frame are $72 \mathrm{Myr}$ and $193 \mathrm{Myr}$, respectively. (b) High surface temperature $\left(T_{0}=1.2\right)$. Here time intervals are $44 \mathrm{Myr}$ and $230 \mathrm{Myr}$. 

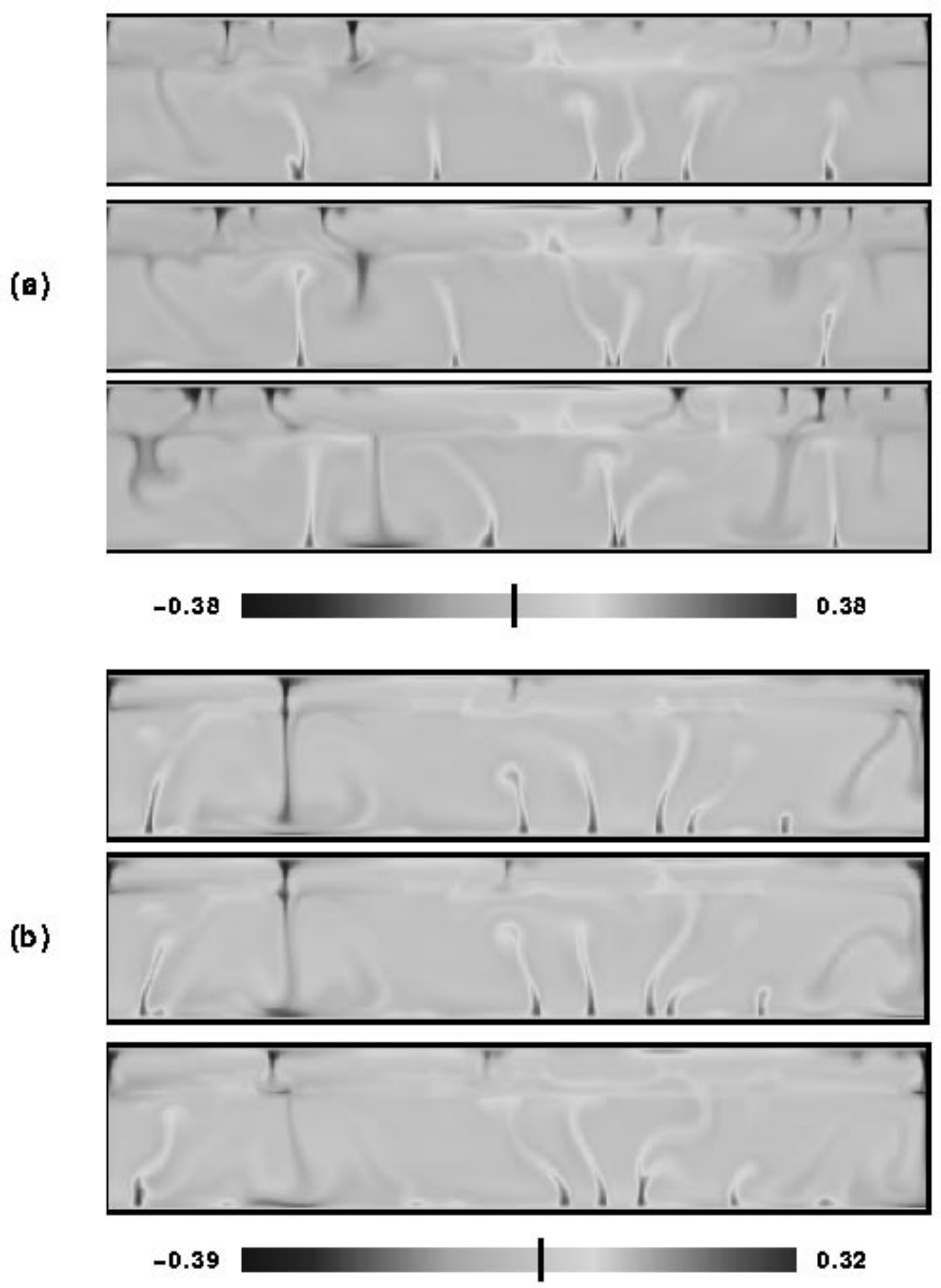

Fig. 2. Snapshots of the residual temperature $\delta T=T-\langle T\rangle$ in the later stage of integration for the cases shown in Fig. 1. (a) $T_{0}=0.2$, time intervals between second and first and between third and first frame are $31 \mathrm{Myr}$ and $66 \mathrm{Myr}$, respectively. (b) $T_{0}=1.2$, time intervals are $20 \mathrm{Myr}$ and $42 \mathrm{Myr}$. 

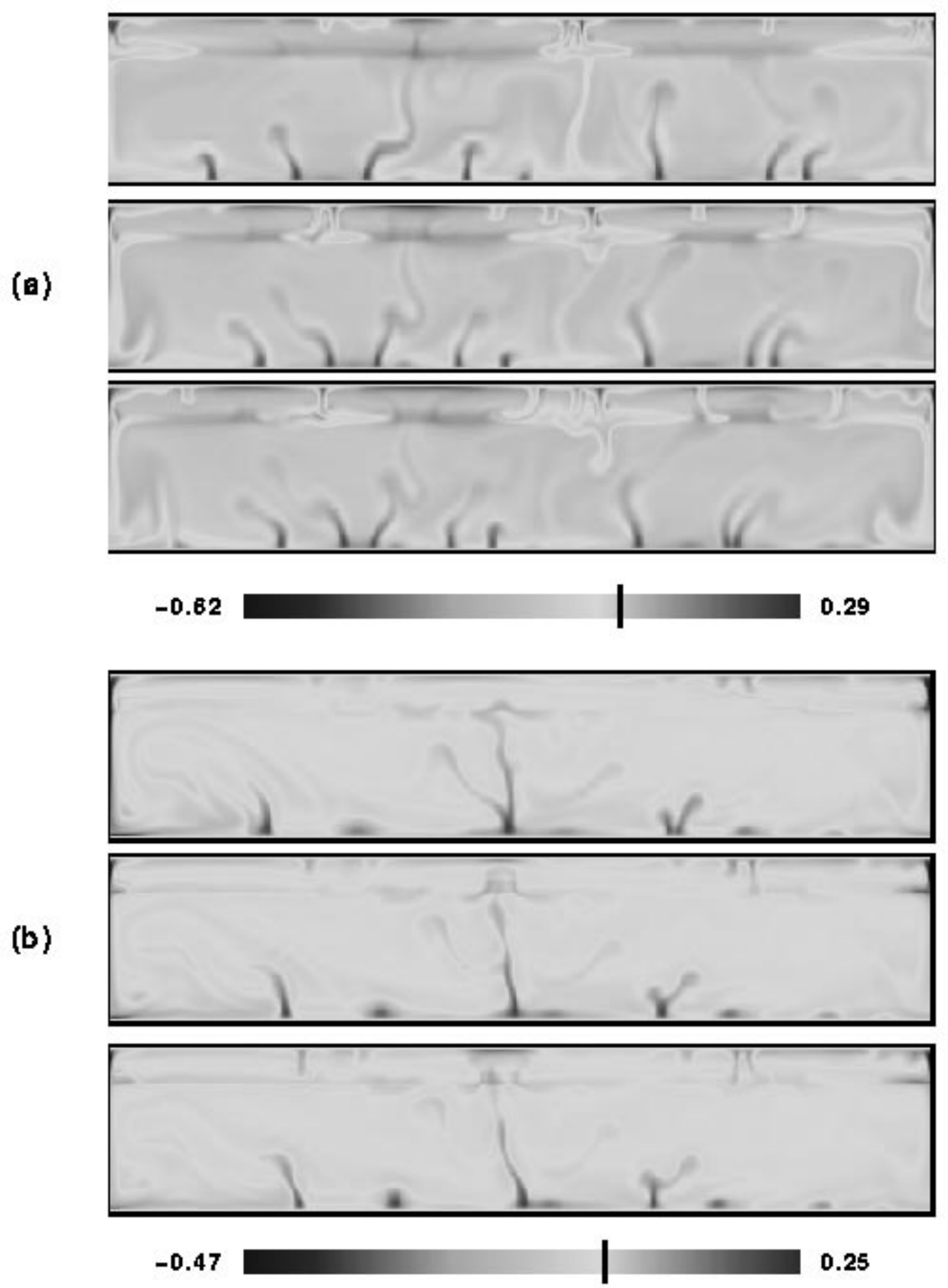

Fig. 3. Same as Fig. 2, now for the also internally heated flows $(R=10)$. (a) $T_{0}=0.2$, time intervals are $44 \mathrm{Myr}$ and $70 \mathrm{Myr}$. (b) $T_{0}=1.2$, time intervals are $15 \mathrm{Myr}$ and $35 \mathrm{Myr}$. 


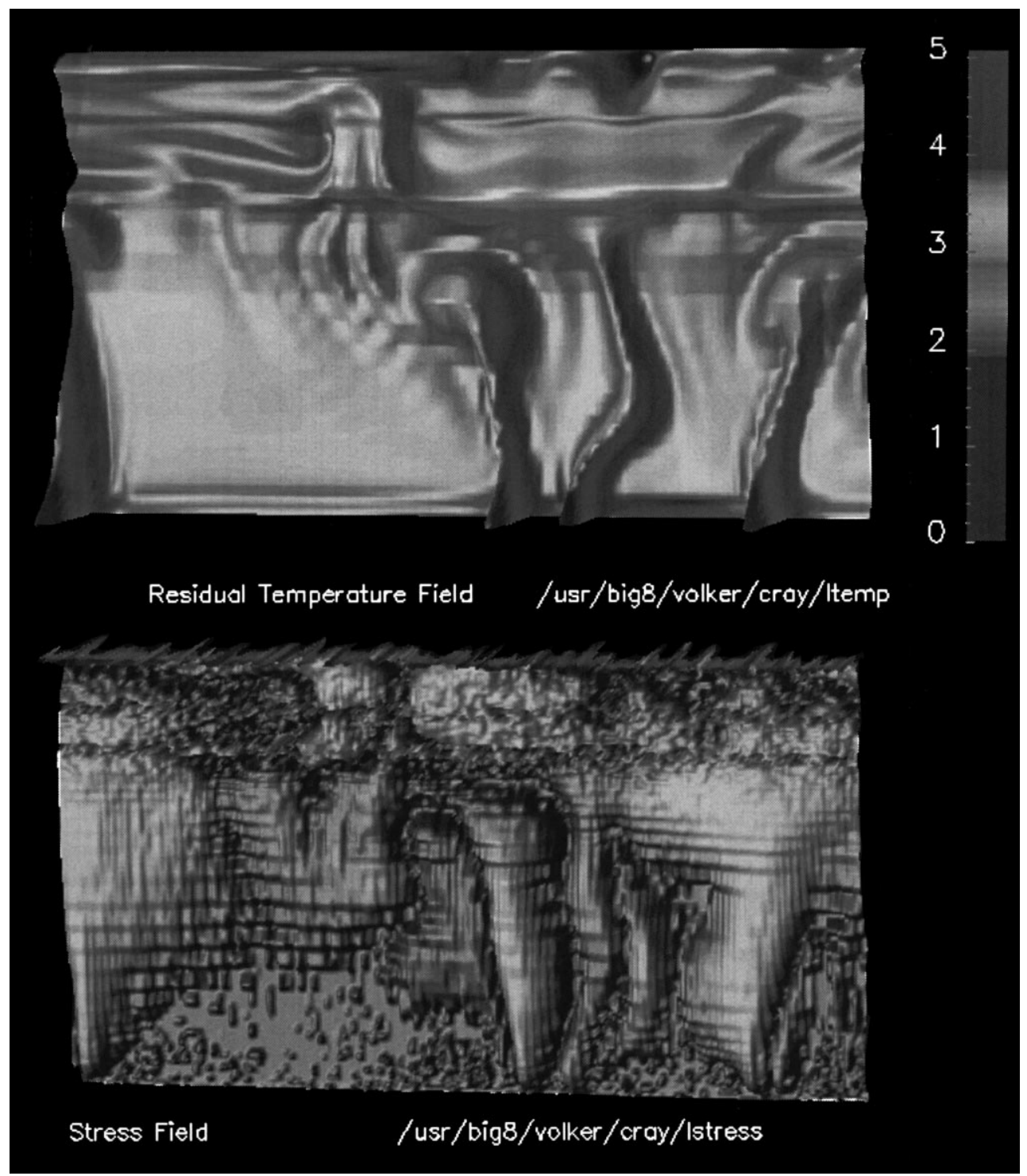

Fig. 4. Close-up shots of (a) the residual temperature taken from the top panel of Fig. 2(a) $\left(T_{0}=0.2\right.$, base heated) and (b) the corresponding shear stress field $\log \left|\tau_{x z}\right|$. The maximum corresponds to a value of $\left|\tau_{x z}\right|=10^{5}$. Stress has been non-dimensionalized in the same manner as for the velocity.

lies like the one shown here are frequently observed in both low and high- $T_{0}$ cases. The stress fields reveal rather complex structures with large magnitudes associated with the upwellings. The stress fields appear similar for both high and low- $T_{0}$ cases. It is interesting to note that the high strain-rates of the rapid upwellings more than compensate the much lower viscosity by producing very high stresses in the lower-mantle upwellings, which have as high stress as the cold downwellings (Fig. 4b). The regions of active upwellings in the upper mantle are also associated with large shear stresses, although shear stress reduction can be observed at the site where the hot material has spread horizontally near the surface (Fig. 4a, upper left-hand corner). 


\section{Discussion and conclusions}

This study has focussed on marshalling evidence for the different aspects of mantle upwellings that occur with high and low surface temperatures in temperature-dependent viscosity convection with two phase transitions. Our results show that in the transient regime for high surface temperature, collective instabilities in the bottom boundary layer can merge together to form a megaplume which can gush forth into the upper mantle and may cause massive resurfacing of the lithosphere. Megaplume injection into the upper mantle is reminiscent of the interaction between the upwellings and the compositional layer in thermal-chemical convection [23]. In the short-time regime at low $T_{0}$ the upwellings are more passive and have return flow in the shallow lower mantle.

In the long time regime the differences in the interaction of upwellings with the transition zone change their character with $T_{0}$. These results are summarized in Fig. 5. The connection between the upwelling and the deep mantle is much better established for the high- $T_{0}$ (Venus-like) than for the low- $T_{0}$ (Earth-like) situation. The return flow in the low- $T_{0}$ case takes place now in the shallow lower mantle with diapiric flow issuing from a deep mantle source. In the high- $T_{0}$ situation there are two types of flow, one involving again a shallow lower-mantle character, while the second one is rising continuously from the core-mantle boundary.

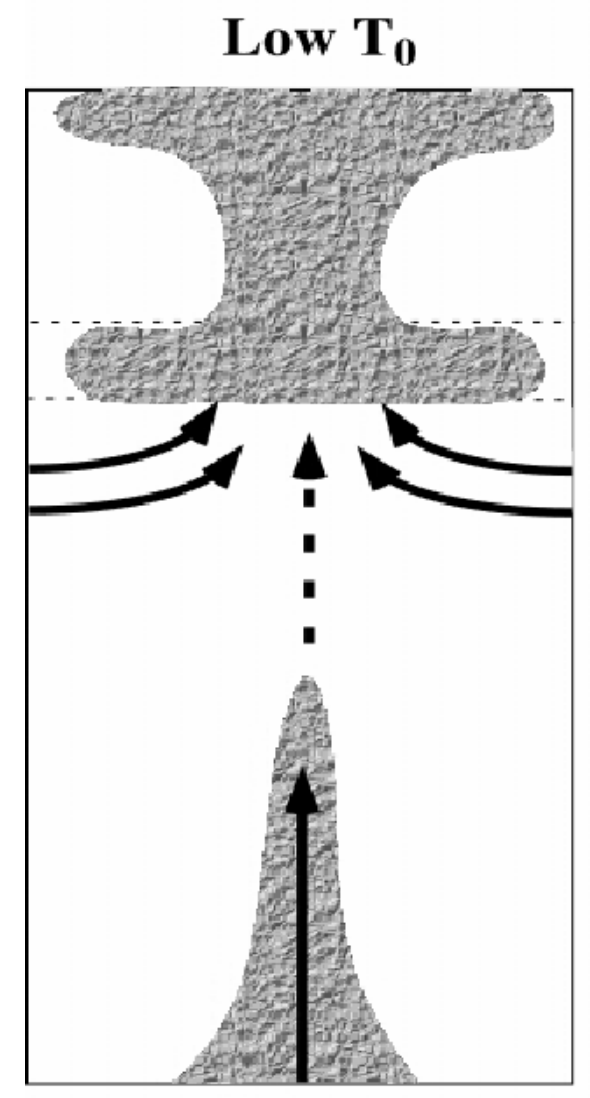

Stage A

\section{$\operatorname{High} \mathbf{T}_{\mathbf{0}}$}

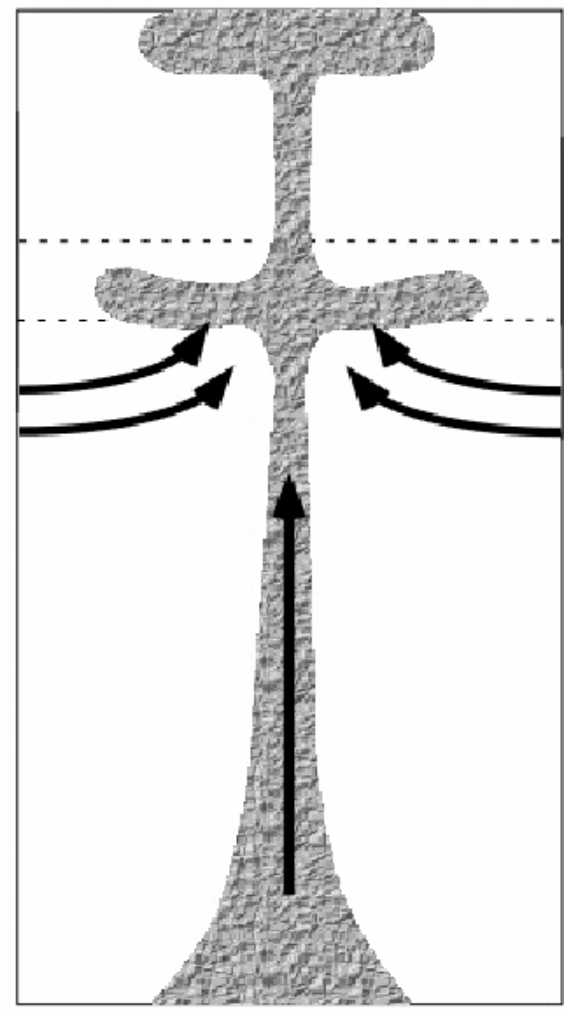

Fig. 5. Sketch of typical plumes in the long-term stage for low (left) and high (right) surface temperature regime. 
Another interesting dynamical effect of high $T_{0}$ is the potential positive feedback mechanism brought on by the massive discharge of hot lower-mantle material into the upper mantle. The hot fluid becomes trapped between a relatively thick lithosphere and the transition zone in a intermittently layered convective situation. Such a scenario, as shown in Fig. 1, may have helped to resurface the Venusian lithosphere in the past $[20,21,24]$, although the effect of high $T_{0}$ may be a minor one, since the massive upwelling of lower mantle material is also observed at lower $T_{0}$. The strength of plumes diminishes with larger amounts of adiabatic cooling, which is proportional to $T_{0}$ [25]. Thus, one can explain why in the low- $T_{0}$ cases there are many boundary-layer-instabilities that develop into plumes rising into the lower mantle but that do not have the strength to penetrate the transition zone. In the high- $T_{0}$ case, many of those instabilities are suppressed before they can form plumes. Instead, they are swept horizontally to feed into the only coherent lower mantle plume, which thus gathers enough buoyancy to penetrate the phase transition region occasionally. This effect of plume concentration would even be stronger with a pressure-dependent viscosity $[26,27]$. The tendency towards fewer, deeper-seated plumes at high $T_{0}$ is in accordance with data from the Magellan mission, which suggest that there are a few large volcanic swells on Venus [28]. Those swells seem to be supported by plumes with a deep-seated mantle origin [29]. In the case of Mars, the giant upwelling has a different dynamical origin. It is due to the plumeplume collision process induced by the fact that the phase transitions of Mars are so close to the Martian core-mantle boundary [10].

From a geochemical standpoint these results are especially intriguing, since the source of mantle plumes may be influenced significantly by the surface temperature of the planet. Current geochemical models (e.g. [30]) seem to favor scenarios with both shallow and deep lower mantle sources of ocean island basalts, while the simulations presented here suggest mostly shallow sources. A temperature- and pressure-dependent rheology, however, would tend to stabilize some plumes from the CMB and thus provide for isolated deep mantle sources. This simple 2-D model yields intuition into how mantle upwellings can be influenced by non-Boussinesq ef- fects, not taken into account in laboratory [31] and numerical models [32,33] of plumes. Non-Newtonian rheology [34] will also change the timescales of this Newtonian model. It should again be mentioned here that in this study we wanted to isolate the effects of $T_{0}$ and therefore kept all other parameters the same. A comparison of Earth and Venus should of course include several other differences between the planets, e.g. rheology and upper (no-slip) boundary condition. Speculations about how these differences would alter the results are beyond the aim of this paper. Even within the parameter space of $T_{0}$ covering Venus and the Earth, large differences have been found. Further studies are needed to test the conclusions and implications of this work, especially for temperature-and pressure-dependent rheology [27]. The question posed here is clear enough.

\section{Acknowledgements}

We thank discussions with David Brunet and Doris Breuer. We also thank T. Ratcliff and A. Lenardic for helpful reviews. V. Steinbach was supported by a M.S.I. travel award. This research has been supported by the geophysics program of the National Science Foundation. [RV]

\section{References}

[1] W.R. Peltier, Penetrative convection in the planetary mantle, Geophys. Fluid Dyn. 5 (1973) 47-88.

[2] V. Steinbach, Numerische Experimente zur Konvektion in kompressiblen Medien, Mitteilungen aus dem Institut fuer Geophysik und Meteorologie, Universitaet zu Koeln, Heft 79, 1991.

[3] D. Bercovici, G. Schubert, G.A. Glatzmaier, Three-dimensional convection of an infinite Prandtl-number compressible fluid in a basally heated spherical shell, J. Fluid Mech. 239 (1992) 683-719.

[4] U. Hansen, D.A. Yuen, Different consequences from compressibility on thermal and thermal-chemical convection in the Earth's outer core, Eos Trans. AGU 76 (17) (1995) 280-281.

[5] D.A. Yuen, S. Balachandar, U. Hansen, Modelling mantle convection: A significant challenge in geophysical fluid dynamics, to P. Fox, R. Kerr (Eds.), Convection, Gordon and Breach, 1998, in press.

[6] G.T. Jarvis, D.P. McKenzie, Convection in a compressible fluid with infinite Prandtl number, J. Fluid Mech. 96 (1980) $515-583$ 
[7] U.R. Christensen, D.A. Yuen, Layered convection induced by phase transitions, J. Geophys. Res. 90 (1985) 1029110300.

[8] V. Steinbach, U. Hansen, A. Ebel, Compressible convection in the Earth's mantle: a comparison of different approaches, Geophys. Res. Lett. 16 (1989) 633-635.

[9] A. Leitch, D.A. Yuen, Internal heating and thermal constraints on the mantle, Geophys. Res. Lett. 16 (1989) 1407-1410.

[10] D. Breuer, D.A. Yuen, T. Spohn, Phase transitions in the Martian mantle: Implications for partially layered convection, Earth Planet. Sci. Lett. 148 (1997) 457-469.

[11] V. Steinbach, D.A. Yuen, Effects of depth-dependent properties on the thermal anomalies produced in flush instabilities from phase-transitions, Phys. Earth Planet. Int. 86 (1994) 165-183.

[12] A. Chopelas, R. Boehler, Thermal expansivity in the lower mantle, Geophys. Res. Lett. 19 (1992) 1983-1986.

[13] W. Zhao, D.A. Yuen, S. Honda, Multiple phase transitions and the style of mantle convection, Phys. Earth Planet. Inter. 72 (1992) 185-210.

[14] V. Steinbach, D.A. Yuen, W. Zhao, Instabilities from phase transitions and the timescales of mantle thermal evolution, Geophys. Res. Lett. 20 (1993) 1119-1122.

[15] D.A. Yuen, D.M. Reuteler, S. Balachandar, V. Steinbach, A.V. Malev-sky, J.J. Smedsmo, Various influences on three-dimensional mantle convection with phase transitions, Phys. Earth Planet. Inter. 86 (1994) 185-203.

[16] L.P. Solheim, W.R. Peltier, Phase boundary deflections at $660 \mathrm{~km}$ depth and episodically layered isochemical convection in the mantle, J. Geophys. Res. 99 (1994) 15861-15875.

[17] P.J. Tackley, On the penetration of an endothermic phase transition by upwellings and downwellings, J. Geophys. Res. 100 (B8) (1995) 15477-15488.

[18] V. Steinbach, D.A. Yuen, The effects of temperature-dependent viscosity on mantle convection with the two major phase transitions, Phys. Earth Planet. Inter. 90 (1995) $13-36$.

[19] P.F. Thompson, P.J. Tackley, Generation of mega-plumes from the core-mantle boundary in a compressible mantle with temperature-dependent viscosity, Geophys. Res. Lett 25 (1998) 1999-2002.

[20] R.G. Strom, G.G. Schaber, D.D. Dawson, The global resurfacing of Venus, J. Geophys. Res. 99 (1994) 10899-10926.
[21] A.T. Basilevsky, J.W. Head, G.G. Schaber, R.G. Strom, The resurfacing history of Venus, in: S.W. Bougher, D.M. Hunten, R.J. Phillips (Eds.), Venus II, Univ. Arizona Press, Tucson, 1997, pp. 1245-1287.

[22] J.T. Ratcliff, D. Bercovici, G. Schubert, L.W. Kroenke, Mantle plume-heads and the initiation of plate tectonic reorganizations, Earth Planet. Sci. Lett. 156 (1998) 195-207.

[23] U. Hansen, D.A. Yuen, Effects of depth-dependent thermal expansivity on the interaction of thermal-chemical plumes with a compositional boundary, Phys. Earth Planet. Inter. 86 (1994) 205-221.

[24] D.L. Turcotte, How does Venus lose heat?, J. Geophys. Res. 100 (E8) (1995) 16931-16940.

[25] W. Zhao, D.A. Yuen, The effects of adiabatic and viscous heatings on plumes, Geophys. Res. Lett. 14 (1987) 1223-1227.

[26] U. Hansen, D.A. Yuen, S.E. Kroening, T.B. Larsen, Dynamical consequences of depth-dependent thermal expansivity and viscosity on mantle circulations and thermal structure, Phys. Earth. Planet. Inter. 77 (1993) 205-223.

[27] V. Steinbach, D.A. Yuen, Dynamical effects of a temperature- and pressure-dependent lower-mantle rheology on the interaction of upwellings with the transition zone, Phys. Earth Planet. Inter. 103 (1997) 85-100.

[28] S.E. Smrekar, W.S. Kiefer, E.R. Stofan, Large volcanic rises on Venus, in: S.W. Bougher, D.M. Hunten, R.J. Phillips (Eds.), Venus II, University of Arizona Press, Tucson, 1997, pp. 845-878.

[29] R.J. Phillips, V.L. Hansen, Geological evolution of Venus: Rises, plains, plumes, and plateaus, Science 279 (1998) 1492-1497.

[30] A.W. Hofmann, Mantle geochemistry: The message from oceanic volcanism, Science 385 (1997) 219-229.

[31] P.L. Olson, , H.A. Singer, Creeping plumesJ. Fluid Mech. 158 (1985) 511-538.

[32] U.R. Christensen, Instability of a hot boundary layer and initiation of thermo-chemical plumes, Annal. Geophys. 2 (1984) 311-320.

[33] P.E. van Keken, Evolution of starting mantle plumes: a comparison between numerical and laboratory models, Earth Planet. Sci. Lett. 148 (1997) 1-11.

[34] T.B. Larsen, D.A. Yuen, Fast plumeheads: Temperaturedependent versus non-Newtonian rheology, Geophys. Res. Lett. 24 (1997) 1995-1998. 